\title{
EVALUATION OF COMPRESSIVE MYELOPATHY BY USING MRI AS THE MODALITY OF CHOICE
}

\section{Radiodiagnosis}

Dr.Nandam Hema Post graduate, Department of Radiodiagnosis, Alluri Sita Ramaraju Academy of Medical Mohana Lakshmi* Sciences, Eluru, A.P.-534005. *Corresponding Author

Dr. S.

Professor and HOD,Department of Radiodiagnosis, Alluri Sita Ramaraju Academy of Venkateswara Rao

Medical Sciences, Eluru, A.P.-534005.

\section{ABSTRACT}

The primary purpose of the study is to discuss the causes of compressive myelopathy, characterize compressive lesions, and to localize the lesion either to extramedullary or intramedullary compartment using magnetic resonance imaging (MRI) as the modality of choice. A hundred patients who came to the hospital and are clinically suspected of having compressive myelopathy underwent MRI. The leading common cause of compressive myelopathy in this study was found to be extradural compression over the cord due to degenerative changes (50\%). The second most common cause was found to be infectious spondylitis (20\%), third being post-traumatic compressive myelopathy (12\%), followed by primary neoplasms \& metastases $(12 \%)$ and other causes like hindbrain and craniovertebral abnormalities $(6 \%)$.

MRI detected cord signal changes in cases with cord compression and also assessed the integrity of the spinal cord, intervertebral discs, and ligament after acute spinal trauma. MRI, though expensive, is a non-invasive, radiation-free modality with high sensitivity, specificity, and accuracy for the evaluation of compressive myelopathy.

\section{KEYWORDS}

compressive myelopathy, intramedullary, extramedullary, extradural compression.

\section{INTRODUCTION}

The term compressive myelopathy refers to a set of pathologic conditions that cause compression over the spinal cord or meninges, leading to damage or dysfunction. The various etiologies for compression may be due to herniation of disc secondary to degenerative changes, posterior vertebral body osteophytes, degenerative changes at Luschka, facet joints, hypertrophy of ligamentum flavum or post-traumatic compression by vertebral fracture, epidural space hemorrhage or infectious collection (infectious spondylodiscitis) or neoplasm or other causes like vascular malformations extradural/intradural (extramedullary), developmental craniovertebral junction abnormalities. These etiological varieties often have ruinous consequences, ranging from quadriplegia and paraplegia to severe sensory deficits. However, many of these are undoubtedly reversible when recognized and treated at an early stage; thus, they are considered amongst the most crucial neuro-medical emergencies. Therefore it is necessary to understand the importance of magnetic resonance imaging when dealing with a multifactorial disease, like compressive myelopathy, for which prognosis depends on early and accurate diagnosis.

\section{OBJECTIVES}

1. To evaluate various causes of compressive myelopathy.

2. MR characterization of spinal cord compressive lesions.

3. To categorize the causes of compression (extradural /intradural extramedullary).

4. MRI in the evaluation of the age and sex distribution of various causes of compressive myelopathy.

\section{MATERIALS AND METHODS}

The study was conducted at the Department of Radiodiagnosis, ALLURI SITA RAMARAJU INSTITUTE OF MEDICAL SCIENCES, West Godavari district. The data necessary for the study was collected from patients with clinical suspicion of compressive myelopathy and were subjected to MRI examination using 1.5 Tesla MRI seimens machine. The study group had a sample size of 100 patients.

\section{RESULTS}

Majority of patients with degenerative, infectious \& post-traumatic myelopathy and primary neoplasms belong to the middle age group (31-50 years). While those patients with metastases belonged to the older age group ( $>50$ years), and others, which included congenital hindbrain and developmental craniovertebral junction abnormalities, belonged to the pediatric \& young adult age group.

Primary neoplasms are more common in the female population, while the majority of the cases of compressive myelopathy associated with the rest of the causes are more common in the male population.

Amongst the various causes of compressive myelopathy, the most common cause in this study was compression due to degenerative changes $(50 \%)$ followed by spinal infection (20\%). Extradural compressive lesions $(92 \%)$ are the most common causes of compressive myelopathy in the current study.

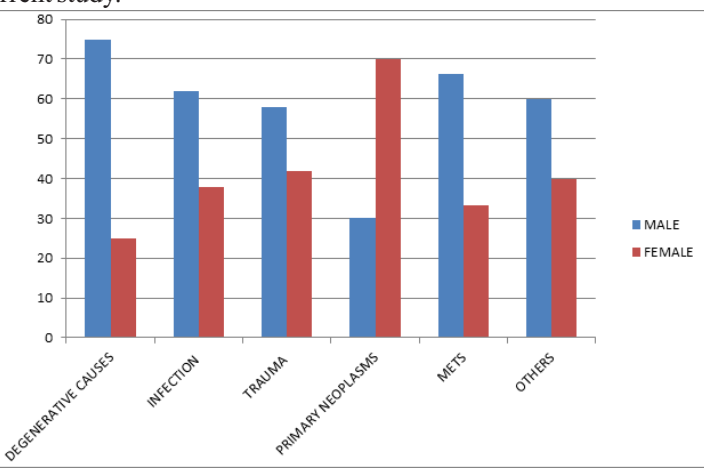

Chart 1: Sex Distribution Of Various Causes Of Compressive Myelopathy.

Table 1: Various Causes Of Compressive Myelopathy.

\begin{tabular}{|l|l|l|}
\hline MRI Diagnosis & $\begin{array}{l}\text { No. Patients } \\
(\mathbf{n = 1 0 0})\end{array}$ & $\mathbf{\%}$ \\
\hline Degenerative causes & 50 & $50 \%$ \\
\hline Infectious spondylitis & 20 & $20 \%$ \\
\hline Trauma & 12 & $12 \%$ \\
\hline Tumors/Mets & 12 & $12 \%$ \\
\hline $\begin{array}{l}\text { Congenital Hindbrain \& } \\
\text { Developmental CVJ abnormalities }\end{array}$ & 6 & $6 \%$ \\
\hline Total & $\mathbf{1 0 0}$ & $\mathbf{1 0 0 \%}$ \\
\hline
\end{tabular}

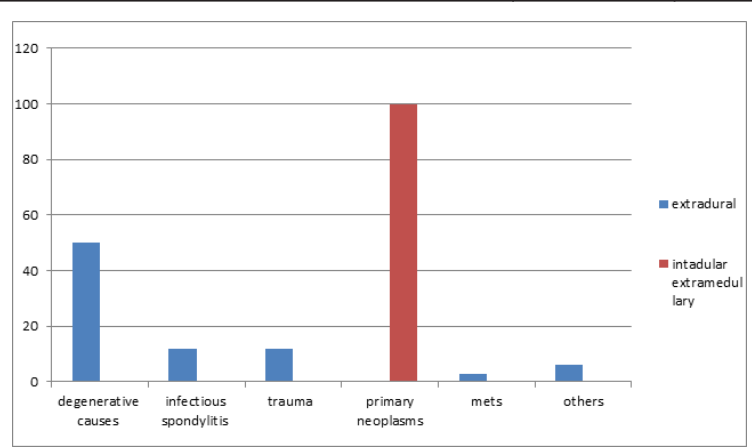


Primary neoplasms are more common in intradural extramedullary compartment while the majority of the cases of compressive myelopathy, i.e., Degenerative causes, infectious spondylitis, and trauma, are common causes for extradural compression in our study.

In spinal injury, the most common site is the thoracic region, followed by the cervical region.

MRI depicts not only the spinal cord changes but also the relationship of subluxated / dislocated vertebral bodies to the cord, posterior elements fracture, ligamentous disruption, soft tissue injuries. All these have a role in prognostic implication, management and can be used to classify damage into stable/unstable.

\section{DISCUSSION}

Off late MRI has been recognized to have better ability in demonstrating the spinal cord with higher sensitivity and specificity and hence is well established as a tool of imaging for trauma, neoplastic, congenital, and degenerative disorders. MRI has become the imaging modality of choice for the spine and spinal cord pathologies due to its multiplanar capabilities, non-invasiveness, and better soft tissue characterization.

In this study, which comprises of 100 cases of compressive myelopathy, we could find varied etiologies for the cause of the compression. Of the 100 cases, 50cases were of degenerative compressive myelopathy ( 40 patients had cervical spondylosis and the rest 10 had lumbar spondylosis), 20 cases were of infectious spondylitis, 12 cases were post-traumatic compressive myelopathy, 12 cases comprising of primary neoplasms \& metastases, and the rest of the 6 cases include congenital hindbrain and developmental craniovertebral junction abnormalities.

In this study, 40 patients( out of 50 cases of degenerative compressive myelopathy) had cervical spondylosis; among them, 34 cases showed cord changes, i.e., intramedullary high signal intensity on $\mathrm{T} 2$ weighted images. Al-Mefty et al. . ' have concluded that high T2 signal reflected myelomalacia or cord gliosis. And, low signal T1 can be related to cystic necrosis and syrinx. Both of which are secondary to longstanding compression of the spinal cord.

Hui Chen et $\mathrm{al}^{2}$. in the year 2016, conducted a study which concluded that the surgical outcomes were more miserable in the patients with both T2 and T1 intramedullary signal changes compared with those without intramedullary signal changes. Thus, Preoperative magnetic resonance imaging can be used as a predictor for postoperative recovery in cervical spondylosis myelopathy patients.

In our study, out of 20 cases of infectious spondylitis, 14 cases were of tuberculous spondylitis, and 6 were pyogenic spondylitis. In cases of TB Spondylitis, MRI showed vertebral body destruction with pre and paravertebral collection and rim enhancement around the intraosseous and paraspinal soft tissue abscesses. In these cases, there is sparing of intervertebral disc space heights, with invariable loss of cortical definition of affected vertebrae in contradistinction to pyogenic spondylitis. In 10 cases, the lesions were noted in the thoracic region, and in 4 cases, the lesions were in the lumbar region. These findings of our study were in concordance with that of Roos DEA et al ${ }^{3}$ and Galhotra RD et al ${ }^{4}$.

Posterior elements involvement was seen in 5 cases of pyogenic spondylitis but was seen in only 3 cases of TB spondylitis in our study. These findings are comparable to the survey conducted by Na-Young Jung et $\mathrm{al}^{5}$, which concluded that posterior elements involvement is common in pyogenic spondylitis but rare in TB spondylitis.

In our study, the level of spinal injuries among the 12 cases of spinal trauma was thoracic $(58.3 \%)$, cervical $(33.3 \%)$, and lumbar $(8.3 \%)$. These findings have correlated with the study conducted by Kerslake et $\mathrm{al}^{6}$. Spinal cord compression was observed in all the 12 cases of spinal injury. The causes of spinal cord compression included vertebral body fracture with retropulsion fracture fragments and epidural hematoma in 8 patients and subluxation of the vertebral body in 4 patients.

In our study, 10 out of 12 traumatic myelopathy patients showed focal hypointensity on T1W and hyperintensity on T2W, STIR images at the level of cord compression, suggestive of cord edema/contusion. These signal changes have a prognostic implication and are in concordance with studies done previously by Hackney et $\mathrm{al}^{7}$. The patients with cord edema recovered entirely or partially, whereas those with hemorrhage in the cord had a poor prognosis.

In our study of 100 cases, there were $3(3 \%)$ cases of metastases causing compressive myelopathy. Among them, one case was due to skeletal metastases from prostatic cancer, and the other case was metastases from an unknown primary. The other was skeletal metastases with its primary in the lung. In all three cases, these spinal lesions were intraspinal extradural in location, causing cord compression. This is in concordance with the study conducted by Lien et al. ." in which $90 \%$ of cases of metastases showed extradural masses.

In our study, all three cases of metastases showed multiplicity. This is in close correlation to the research done by Lien et al ${ }^{8}$, in which $78 \%$ of cases of metastases had multiplicity.

In our study, the level of lesions in 2 cases of metastases is in the thoracic region, and in the third case, lesions were in the cervical area. This is in comparison to the study done by Livingston et al ${ }^{9}$.

Out of 9 cases of primary neoplasms, we had 8 cases of intradural extramedullary neoplasms. These values are highly comparable to the study conducted by Cormick PC et $\mathrm{al}^{10}$, which concluded that $2 / 3 \mathrm{rd}$ of intradural tumors are extramedullary.

In our study, among the 8 cases of intradural extramedullary primary neoplasms, there were 4 cases of meningioma, 3 cases of ependymoma, and a single case of NF II where the lesions were plexiform neurofibromas. Signal intensity changes were seen in all the three cases of meningioma, correlated with a study conducted by Matsumoto $\mathrm{S}$ et a $\mathrm{l}^{11}$.

\section{CONCLUSION}

MRI is the definitive modality in assessing spinal soft tissue injuries, especially in the evaluation of spinal cord edema /contusion, intervertebral discs, and ligaments. MRI is the most sensitive modality to detect, characterize spinal infection, and spinal tumors. However, the final diagnosis still relies on biopsy and culture. To date, MRI is the best modality to image the spinal cord directly. In our study, with the help of MRI, we could successfully detect and characterize the spinal tumor based on location into extradural/ intradural and assess the integrity of the spinal cord, intervertebral discs, and ligament after acute spinal trauma. So, in the end, we conclude that MRI is a very definitive, sensitive, accurate, though costly but highly specific, noninvasive, radiation-free modality for evaluation of compressive myelopathy.

\section{REFERENCES}

Al-Mefty O, Harkey LH, Middleton TH, et al. Myelopathic cervical spondylotic lesions demonstrated by magnetic resonance imaging. J Neurosurg. 1988;68:217-22.

2. Chen H, Pan J, Nisar M, Zeng HB, Dai LF, Lou C, Zhu SP, Dai B, Xiang GH. The value of preoperative magnetic resonance imaging in predicting a postoperative recovery in of preperave patients with cervical

3. Roos DEA, Persijn V, Meerten EL, Bloem JI. MRI of Tubercular Spondylitis: AJR 1986; 146: 79-82.

4. Galhotra RD, Jain T, Sandhu P, Galhotra V. Utility of magnetic resonance imaging in the differential diagnosis of tubercular and pyogenic spondylodiscitis. J Nat Sci Biol Med. 2015 Jul-Dec;6(2):388-93

5. Na-Young Jung, Won-hee Jee, Kee-Yong Ha, Chun-Kun Park, Jae-Young Byun. Discrimination of Tuberculous Spondylitis from Pyogenic Spondylitis on MRI. AJR $2004 \cdot 182: 1405-1410$

6. Kerslake RW, Jaspan T, Worthington BS. Magnetic Resonance Imaging of Spinal Trauma: The British Journal of Radiology. 1991;64:386-402.

7. Hackney DB, Asata R, Sci DM, Joseph PM, Carvlin MJ, McGrath JT, Grossman RI, et al. Hemorrhage and edema in Acute spinal cord compression. Demonstration by MR Imaging: Radiology. 1986; 161:387 -390

8. Lien HH, Blomlie V, Heimdal K. Magnetic Resource Imaging of compression: Acta Radiologica. 1990; 31: 187-190

9. Livingston KE, Perrin RG. The Neurosurgical management of spinal metastases causing cord and cauda equina compression: J. Neurosurg. 1978; 49: 590 -594

10. Cormic PC, Post KD, Stein BM. Intradural Extramedullary tumors in Adults: Neurosurgery clinics of North America. 1990; 1:591-608.

11. Matsumoto S, Hasuo K, Uchino A, Mizushima A, Kawa, Matsuura Y, et al MRI of Intradural Extramedullary Spinal neurinomas and meningiomas: Clinical Imaging. $1993 ; 17: 46-52$ 